\title{
Parity nonconservation in Fr-like actinide and Cs-like rare-earth ions
}

\author{
B. M. Roberts ${ }^{*}$ V. A. Dzuba, and V. V. Flambaum \\ School of Physics, University of New South Wales, Sydney, NSW 2052, Australia
}

(Dated: November 20, 2018)

\begin{abstract}
Parity nonconservation amplitudes are calculated for the $7 s-6 d_{3 / 2}$ transitions of the francium isoelectronic sequence ( $\mathrm{Fr}, \mathrm{Ra}^{+}, \mathrm{Ac}^{2+}, \mathrm{Th}^{3+}, \mathrm{Pa}^{4+}, \mathrm{U}^{5+}$ and $\mathrm{Np}^{6+}$ ) and for the $6 s-5 d_{3 / 2}$ transitions of the cesium isoelectronic sequence $\left(\mathrm{Cs}, \mathrm{Ba}^{+}, \mathrm{La}^{2+}, \mathrm{Ce}^{3+}\right.$ and $\left.\mathrm{Pr}^{4+}\right)$. We show in particular that isotopes of $\mathrm{La}^{2+}, \mathrm{Ac}^{2+}$ and $\mathrm{Th}^{3+}$ ions have strong potential in the search for new physics beyond the standard model - the PNC amplitudes are large, the calculations are accurate and the nuclei are practically stable. In addition, ${ }^{232} \mathrm{Th}^{3+}$ ions have recently been trapped and cooled [C. J. Campbell et al., Phys. Rev. Lett. 102, 233004 (2009)]. We also extend previous works by calculating the $s$ - $s$ PNC transitions in $\mathrm{Ra}^{+}$and $\mathrm{Ba}^{+}$, and provide new calculations of several energy levels, and electric dipole and quadrupole transition amplitudes for the Fr-like actinide ions.

PACS numbers: 11.30.Er, 31.15.A-, 31.30.jg
\end{abstract}

\section{INTRODUCTION}

The parity nonconservation (PNC) amplitude of the $6 s-7 s$ transition in cesium is presently the most precise low-energy test of the electroweak theory. This precision is a result of highly accurate measurements [1] and the almost equally accurate atomic calculations used for their interpretation 2 [5]. This interpretation shows that the value of the weak nuclear charge for ${ }^{133} \mathrm{Cs}$, coming from the PNC measurements, differs from the prediction of the standard model by $1.6 \sigma$ [2, 6]. Although this cannot be regarded as disagreement, it indicates that further improvements to the accuracy of the measurements and interpretation may lead to new important results.

Additionally, the need for new measurements is motivated by the requirement to check the existing results. It is very important that an independent test of the existing experimental results [1] is performed. These are very important results and must be checked even if the accuracy is not improved. Moreover, there is a good chance for both theoretical and experimental improvement since the PNC amplitudes of these systems are up to 50 times larger than in Cs.

It is natural to expect a higher accuracy in measurements of systems where the PNC effect is larger. On the other hand, for high accuracy of the calculations it is important to have systems with a simple electron structure. The $s$ - $d$ transitions in the Fr-like ions considered in this paper seem to be very promising in this regard. The PNC amplitude is larger for atoms or ions with higher nuclear charge $Z$ [7. It is also larger for the $s$ - $d$ transitions 8] than for the $s$ - $s$ transitions like the one used in cesium 2. The accuracy of these calculations is also expected to be high since the ions have simple electron structure with one valence electron above closed shells similar to that of cesium.

* b.roberts@unsw.edu.au
There are several additional factors which promise potentially better theoretical accuracy for these ions than for Cs:

- The main source of theoretical uncertainty is electron correlations. The relative value of the correlation correction is smaller for ions than for neutral atoms.

- There are no strong cancellations between different correlation corrections for $s-d$ PNC amplitudes 8 in contrast to very strong cancellation for the $6 s-7 s$ PNC amplitude in Cs.

- The $s-d$ PNC amplitude is strongly dominated by the term with the $d-p$ electric dipole transition amplitude and $s-p$ weak matrix element. This term can be checked and/or corrected if an accurate experimental value for the $d-p$ amplitude is known. A similar approach in Cs works with limited accuracy due to strong cancellation between $6 s-n p$ and $7 s-n p$ contributions.

PNC measurements have been considered for the $\mathrm{Ba}^{+}$ ion 9 and are in progress for the $\mathrm{Ra}^{+}$ion [10. The FrPNC collaboration has begun the construction of a laser cooling and trapping apparatus with the purpose of measuring atomic parity nonconservation in microwave and optical transitions of francium 11. With a PNC amplitude in the $7 s-8 s$ optical transition expected to be around 15 times larger than that of cesium, and its relatively simple electronic configuration, francium is a very good candidate atom for precision measurements and calculations of PNC [8, 12, 13 .

With the aim of motivating experiment in this important area, we present calculations of $s-s$ and $s-d$ PNC amplitudes for several Cs- and Fr-like ions. Simple estimates show that the size of the PNC effect should scale as $E_{P N C} \sim Z^{3} R(Z \alpha) / Z_{a}$, where $R$ is a relativistic factor, $Z$ is nuclear charge and $Z_{a}$ is the effective charge defined as $E_{n}=-Z_{a}^{2} / 2 n^{2}$ atomic units [14. Here, $E_{n}$ is the energy of valence electron, $n$ the principal quantum 
number ( $n=7$ for Fr-like ions). Therefore PNC effects in these ions are only slightly smaller than in neutral atoms.

Of particular interest are the optical $s-d$ PNC transitions of ${ }^{232} \mathrm{Th}^{3+}$ and ${ }^{139} \mathrm{La}^{2+}$, and the IR transition in ${ }^{227} \mathrm{Ac}^{2+} .{ }^{232} \mathrm{Th}$ has a half-life of $1.5 \times 10^{10}$ years and ${ }^{227} \mathrm{Ac}$ of 21.8 years, much more stable than Fr with its most stable isotope $\left({ }^{223} \mathrm{Fr}\right)$ having a half-life of just 22 minutes. ${ }^{139} \mathrm{La}^{2+}$ is stable. Importantly, the ${ }^{232} \mathrm{Th}^{3+}$ ion has been trapped and cooled by Campbell et al. [15]. This was the first reported laser cooling of a multiply charged ion.

The experiment needed to measure the $7 s-6 d_{3 / 2}$ PNC amplitude in $\mathrm{Th}^{3+}$ is somewhat different to the conventional PNC experiments. Neither of the states of interest are the ground state and the PNC amplitude must be reached by first populating the metastable $7 s$ state. This is explored in more detail in the later sections. $\mathrm{Ac}^{2+}$ maintains a $7 s$ ground state, and has a very long lived $6 d_{3 / 2}$ state, which is highly beneficial for PNC measurements [9].

${ }^{227} \mathrm{Ac}$ and the odd-nucleon isotope ${ }^{229} \mathrm{Th}$ (with a halflife of 7340 years) will also be of interest for measurements of nuclear-spin-dependent PNC in optical or hyperfine transitions, including the extraction of the nuclear anapole moment and the strength of the PNC nuclear forces (see, e.g. [16]).

\section{CALCULATIONS}

The PNC amplitude of a transition between states $a$ and $b$ of the same parity can be expressed via the sum over opposite parity states $n$,

$$
E_{P N C}=\sum_{n}\left[\frac{\left\langle b\left|\hat{d}_{E 1}\right| n\right\rangle\left\langle n\left|\hat{h}_{W}\right| a\right\rangle}{E_{a}-E_{n}}+\frac{\left\langle b\left|\hat{h}_{W}\right| n\right\rangle\left\langle n\left|\hat{d}_{E 1}\right| a\right\rangle}{E_{b}-E_{n}}\right],
$$

where $a, b$, and $n$ are many-electron wave functions of the atom, $\hat{d}_{E 1}$ is the electric dipole transition operator and $\hat{h}_{W}=\frac{G_{F}}{2 \sqrt{2}} Q_{W} \rho(r) \gamma_{5}$ is the nuclear-spin-independent PNC interaction $\left(G_{F}\right.$ the Fermi constant, $Q_{W}$ is the nuclear weak charge, $\rho$ the nucleon density and the Dirac matrix $\left.\gamma_{5}=i \gamma_{0} \gamma_{1} \gamma_{2} \gamma_{3}\right)$.

The exact expression (1) can be reduced to an approximate one which looks very similar but contains singleelectron matrix elements and energies. This serves as a base for the sum-over-states calculations. Many-body effects are included in this approach by modifying singleelectron orbitals and the external field operators. We use this approach for tests only. The actual calculations are done using a different approach based on solving differential equations. This approach has many important advantages which will be discussed below.

We start from the mean-field Dirac-Fock approximation with a $V^{N-1}$ potential, which is particularly convenient for the single valence electron ions studied here, and then include dominating electron correlation effects. Core-valence correlation corrections to the valence elec- tron wavefunction are included via the correlation potential method [17. The correlation potential, $\hat{\Sigma}$, including summation of the series of dominating diagrams, is calculated in all orders of perturbation theory using relativistic Green functions and the Feynman diagram technique [18. The correlation potential $\hat{\Sigma}$ is then used to construct the so-called Brueckner orbitals (BOs) for the valence electron. The BOs are found by solving the relativistic Hartree-Fock-like equations with the extra operator $\hat{\Sigma}:\left(\hat{H}_{0}+\hat{\Sigma}-E_{n}\right) \psi_{n}^{(\mathrm{BO})}=0$, where $\hat{H}_{0}$ is the relativistic Hartree-Fock Hamiltonian and the index $n$ denotes valence states. The $\mathrm{BO} \psi_{n}^{(\mathrm{BO})}$ and energy $E_{n}$ include correlations.

The weak and electric dipole interactions and the electron core polarization effects are included via the timedependent Hartree-Fock approximation [17, 18, which is equivalent to the well-known random phase approximation (RPA). The single-electron wavefunction in an external weak and $E 1$ field is expressed in the RPA method as $\psi=\psi_{0}+\delta \psi+X e^{-i \omega t}+Y e^{i \omega t}+\delta X e^{-i \omega t}+\delta Y e^{i \omega t}$, where $\psi_{0}$ is the unperturbed state, $\delta \psi$ is the correction due to weak interaction acting alone, $X$ and $Y$ are corrections due to the photon field acting alone, $\delta X$ and $\delta Y$ are corrections due to both fields acting simultaneously, and $\omega$ is the frequency of the PNC transition. Where possible, $\omega$ should be taken from the experimental energy of the transition. The corrections are found by solving the system of RPA equations self-consistently for the core:

$$
\begin{aligned}
\left(\hat{H}_{0}-E_{c}\right) \delta \psi_{c} & =-\left(\hat{h}_{W}+\delta \hat{V}_{W}\right) \psi_{0 c}, \\
\left(\hat{H}_{0}-E_{c}-\omega\right) X_{c} & =-\left(\hat{d}_{E 1}+\delta \hat{V}_{E 1}\right) \psi_{0 c}, \\
\left(\hat{H}_{0}-E_{c}+\omega\right) Y_{c} & =-\left(\hat{d}_{E 1}^{\dagger}+\delta \hat{V}_{E 1}^{\dagger}\right) \psi_{0 c}, \\
\left(\hat{H}_{0}-E_{c}-\omega\right) \delta X_{c} & =-\delta \hat{V}_{E 1} \delta \psi_{c}-\delta \hat{V}_{W} X_{c}-\delta \hat{V}_{E 1 W} \psi_{0 c}, \\
\left(\hat{H}_{0}-E_{c}+\omega\right) \delta Y_{c} & =-\delta \hat{V}_{E 1}^{\dagger} \delta \psi_{c}-\delta \hat{V}_{W} Y_{c}-\delta \hat{V}_{E 1 W}^{\dagger} \psi_{0 c},
\end{aligned}
$$

where the index $c$ denotes core states, $\delta \hat{V}_{W}$ and $\delta \hat{V}_{E 1}$ are corrections to the core potential arising from the weak and E1 interactions respectively and $\delta \hat{V}_{E 1 W}$ is the correction to the core potential arising from the simultaneous perturbation of the weak field and the electric field of the laser light.

The PNC amplitude between valence states $a$ and $b$ in the RPA approximation is given by

$$
\begin{aligned}
E_{\mathrm{PNC}} & =\left\langle\psi_{b}\left|\hat{d}_{E 1}+\delta \hat{V}_{E 1}\right| \delta \psi_{a}\right\rangle+\left\langle\psi_{b}\left|\hat{h}_{W}+\delta \hat{V}_{W}\right| X_{a}\right\rangle \\
& +\left\langle\psi_{b}\left|\delta \hat{V}_{E 1 W}\right| \psi_{a}\right\rangle \\
& =\left\langle\psi_{b}\left|\hat{d}_{E 1}+\delta \hat{V}_{E 1}\right| \delta \psi_{a}\right\rangle+\left\langle\delta \psi_{b}\left|\hat{d}_{E 1}+\delta \hat{V}_{E 1}\right| \psi_{a}\right\rangle \\
& +\left\langle\psi_{b}\left|\delta \hat{V}_{E 1 W}\right| \psi_{a}\right\rangle .
\end{aligned}
$$

By using BOs for the valence states $\psi_{a}$ and $\psi_{b}$ in (3) we can include correlations in the calculation of the PNC amplitude. The corrections $\delta \psi_{a}$ and $\delta \psi_{b}$ to the BOs $\psi_{a}$ and $\psi_{b}$ are also found with the use of the correlation potential $\hat{\Sigma}$ :

$$
\left(\hat{H}_{0}-E_{a}+\hat{\Sigma}\right) \delta \psi_{a}=-\left(\hat{h}_{W}+\delta \hat{V}\right) \psi_{0 a} .
$$


TABLE I. Comparison of calculated energy levels (BO) and experimental values (Ref. [19]) for $\mathrm{Cs}, \mathrm{Ba}^{+}, \mathrm{Fr}^{\mathrm{and}} \mathrm{Ra}^{+}$. (cm ${ }^{-1}$ )

\begin{tabular}{|c|c|c|c|c|c|c|c|c|c|c|c|}
\hline Level & $\begin{array}{l}\text { Cs } \\
\text { BO }\end{array}$ & Exp. & & $\begin{array}{l}\mathrm{Ba}^{+} \\
\mathrm{BO}\end{array}$ & Exp. & & $\begin{array}{c}\mathrm{Fr} \\
\mathrm{BO}\end{array}$ & Exp. & & $\begin{array}{l}\mathrm{Ra}^{+} \\
\mathrm{BO}\end{array}$ & Exp. \\
\hline $6 s_{1 / 2}$ & 0 & 0 & $6 \mathrm{~s}_{1 / 2}$ & 0 & 0 & $7 \mathrm{~s}_{1 / 2}$ & 0 & 0 & $7 \mathrm{~s}_{1 / 2}$ & 0 & $\overline{0}$ \\
\hline $6 \mathrm{p}_{1 / 2}$ & 11168 & 11178 & $5 \mathrm{~d}_{3 / 2}$ & 4280 & 4874 & $7 \mathrm{p}_{1 / 2}$ & 12218 & 12237 & $6 \mathrm{~d}_{3 / 2}$ & 11741 & 12084 \\
\hline $6 \mathrm{p}_{3 / 2}$ & 11736 & 11732 & $5 d_{5 / 2}$ & 5128 & 5675 & $7 \mathrm{p}_{3 / 2}$ & 13954 & 13924 & $6 d_{5 / 2}$ & 13471 & 13743 \\
\hline $5 d_{3 / 2}$ & 14310 & 14499 & $6 \mathrm{p}_{1 / 2}$ & 20234 & 20262 & $6 d_{3 / 2}$ & 16200 & 16230 & $7 \mathrm{p}_{1 / 2}$ & 21291 & 21351 \\
\hline $5 d_{5 / 2}$ & 14426 & 14597 & $6 \mathrm{p}_{3 / 2}$ & 21960 & 21952 & $6 d_{5 / 2}$ & 16412 & 16430 & $7 \mathrm{p}_{3 / 2}$ & 26259 & 26209 \\
\hline $7 \mathrm{~s}_{1 / 2}$ & 18631 & 18536 & $7 \mathrm{~s}_{1 / 2}$ & 42647 & 42355 & $8 s_{1 / 2}$ & 19862 & 19740 & $8 s_{1 / 2}$ & 43757 & 43405 \\
\hline $7 \mathrm{p}_{1 / 2}$ & 21818 & 21765 & $6 \mathrm{~d}_{3 / 2}$ & 46234 & 45949 & $8 \mathrm{p}_{1 / 2}$ & 23190 & 23113 & $7 \mathrm{~d}_{3 / 2}$ & 49082 & 48744 \\
\hline $7 \mathrm{p}_{3 / 2}$ & 22000 & 21946 & $6 d_{5 / 2}$ & 46438 & 46155 & $8 \mathrm{p}_{3 / 2}$ & 23737 & 23658 & $5 f_{5 / 2}$ & 49254 & 48988 \\
\hline $6 \mathrm{~d}_{3 / 2}$ & 22611 & 22589 & $4 f_{5 / 2}$ & 47829 & 48259 & $7 \mathrm{~d}_{3 / 2}$ & 24311 & 24244 & $7 d_{5 / 2}$ & 49485 & 49240 \\
\hline $6 \mathrm{~d}_{5 / 2}$ & 22656 & 22632 & $4 f_{7 / 2}$ & 48219 & 48483 & $7 \mathrm{~d}_{5 / 2}$ & 24402 & 24333 & $5 f_{7 / 2}$ & 49569 & 49272 \\
\hline $8 s_{1 / 2}$ & 24391 & 24317 & $7 \mathrm{p}_{1 / 2}$ & 49595 & 49390 & $9 \mathrm{~s}_{1 / 2}$ & 25773 & 25671 & $8 \mathrm{p}_{1 / 2}$ & 50864 & 50606 \\
\hline $4 \mathrm{f}_{7 / 2}$ & 24528 & 24472 & $7 \mathrm{p}_{3 / 2}$ & 50213 & 50011 & $5 f_{7 / 2}$ & 25970 & - & $8 p_{3 / 2}$ & 52635 & 52392 \\
\hline $4 f_{5 / 2}$ & 24528 & 24472 & $8 s_{1 / 2}$ & 58258 & 58025 & $5 f_{5 / 2}$ & 25971 & - & $9 \mathrm{~s}_{1 / 2}$ & 59448 & 59165 \\
\hline Lim. ${ }^{a}$ & 31458 & 31406 & & 80838 & 80686 & & 32925 & 32849 & & 82035 & 81842 \\
\hline
\end{tabular}

a Ionization energy of the ground-state valence electron

TABLE II. Calculated energy levels (BO) for the francium-like actinide ions and available experimental data [20]. $\left(\mathrm{cm}^{-1}\right)$

\begin{tabular}{|c|c|c|c|c|c|c|c|c|c|c|c|c|}
\hline \multicolumn{3}{|c|}{$\mathrm{Ac}^{2+}$} & \multicolumn{3}{|c|}{$\mathrm{Th}^{3+}$} & \multicolumn{2}{|c|}{$\mathrm{Pa}^{4+}$} & \multicolumn{3}{|c|}{$\mathrm{U}^{5+}$} & \multicolumn{2}{|c|}{$\mathrm{Np}^{6+}$} \\
\hline Level & $\mathrm{BO}$ & Exp. & & $\mathrm{BO}$ & Exp. & & $\mathrm{BO}$ & & $\mathrm{BO}$ & Exp. & & $\mathrm{BO}$ \\
\hline $7 \mathrm{~s}_{1 / 2}$ & 0 & 0 & $5 f_{5 / 2}$ & 0 & 0 & $5 f_{5 / 2}$ & 0 & $5 f_{5 / 2}$ & 0 & 0 & $5 f_{5 / 2}$ & $\overline{0}$ \\
\hline $6 \mathrm{~d}_{3 / 2}$ & 435 & 801 & $5 \mathrm{f}_{7 / 2}$ & 4393 & 4325 & $5 f_{7 / 2}$ & 6061 & $5 f_{7 / 2}$ & 7784 & 7609 & $5 f_{7 / 2}$ & 9470 \\
\hline $6 \mathrm{~d}_{5 / 2}$ & 3926 & 4204 & $6 \mathrm{~d}_{3 / 2}$ & 8681 & 9193 & $6 \mathrm{~d}_{3 / 2}$ & 48302 & $6 \mathrm{~d}_{3 / 2}$ & 90713 & 91000 & $6 \mathrm{~d}_{3 / 2}$ & 139415 \\
\hline $5 f_{5 / 2}$ & 23467 & 23455 & $6 \mathrm{~d}_{5 / 2}$ & 14084 & 14486 & $6 \mathrm{~d}_{5 / 2}$ & 55753 & $6 \mathrm{~d}_{5 / 2}$ & 100369 & 100511 & $6 \mathrm{~d}_{5 / 2}$ & 151486 \\
\hline $5 f_{7 / 2}$ & 26112 & 26080 & $7 \mathrm{~s}_{1 / 2}$ & 22948 & 23131 & $7 \mathrm{~s}_{1 / 2}$ & 79208 & $7 \mathrm{~s}_{1 / 2}$ & 141157 & 141448 & $7 \mathrm{~s}_{1 / 2}$ & 211402 \\
\hline $7 \mathrm{p}_{1 / 2}$ & 29375 & 29466 & $7 \mathrm{p}_{1 / 2}$ & 59957 & 60239 & $7 \mathrm{p}_{1 / 2}$ & 123396 & $7 \mathrm{p}_{1 / 2}$ & 193744 & 193340 & $7 \mathrm{p}_{1 / 2}$ & 273437 \\
\hline $7 \mathrm{p}_{3 / 2}$ & 38136 & 38063 & $7 \mathrm{p}_{3 / 2}$ & 72995 & 73056 & $7 p_{3 / 2}$ & 141201 & $7 \mathrm{p}_{3 / 2}$ & 216937 & 215886 & $7 p_{3 / 2}$ & 301680 \\
\hline $8 s_{1 / 2}$ & 69660 & & $8 s_{1 / 2}$ & 120357 & 119622 & $7 d_{3 / 2}$ & 201271 & $6 f_{5 / 2}$ & 283289 & & $6 f_{5 / 2}$ & 365542 \\
\hline $7 \mathrm{~d}_{3 / 2}$ & 73543 & & $7 \mathrm{~d}_{3 / 2}$ & 120907 & 119685 & $7 \mathrm{~d}_{5 / 2}$ & 203789 & $6 f_{7 / 2}$ & 284244 & & $6 \mathrm{f}_{7 / 2}$ & 366832 \\
\hline $7 d_{5 / 2}$ & 74579 & & $7 d_{5 / 2}$ & 122622 & 121427 & $6 f_{5 / 2}$ & 203997 & $7 \mathrm{~d}_{3 / 2}$ & 288691 & & $7 \mathrm{~d}_{3 / 2}$ & 382067 \\
\hline $8 \mathrm{p}_{1 / 2}$ & 80612 & & $8 \mathrm{p}_{1 / 2}$ & 135196 & 134517 & $6 \mathrm{f}_{7 / 2}$ & 204665 & $7 \mathrm{~d}_{5 / 2}$ & 292124 & & $7 \mathrm{~d}_{5 / 2}$ & 386532 \\
\hline $6 f_{5 / 2}$ & 83166 & & $8 \mathrm{p}_{3 / 2}$ & 140536 & 139871 & $8 \mathrm{~s}_{1 / 2}$ & 205653 & $8 s_{1 / 2}$ & 299566 & & $8 s_{1 / 2}$ & 400640 \\
\hline $6 f_{7 / 2}$ & 83513 & & $9 \mathrm{~s}_{1 / 2}$ & 161461 & 160728 & $8 \mathrm{p}_{1 / 2}$ & 224369 & $8 \mathrm{p}_{1 / 2}$ & 322245 & & $8 \mathrm{p}_{1 / 2}$ & 428393 \\
\hline Lim.: & 141221 & $140594^{a}$ & & 232015 & $231065^{a}$ & & 363394 & & 509109 & $50000 d^{a}$ & & 667359 \\
\hline
\end{tabular}

a Theoretical values: 140590(160), 231065(200), 500000(13000) [19]

TABLE III. Percentage difference between calculations and experiment for the most important energy intervals for PNC.

\begin{tabular}{rrrrr}
\hline \hline Energy Interval & $\mathrm{Fr}$ & \multicolumn{1}{c}{$\mathrm{Ra}^{+}$} & $\mathrm{Ac}^{2+}$ & $\mathrm{Th}^{3+}$ \\
\hline $7 s_{1 / 2}-7 p_{1 / 2}$ & $-0.2 \%$ & $-0.3 \%$ & $-0.3 \%$ & $-0.3 \%$ \\
$7 s_{1 / 2}-8 p_{1 / 2}$ & $0.3 \%$ & $0.5 \%$ & - & $0.8 \%$ \\
\hline \hline
\end{tabular}

Note that the last term in (3) gives an important contribution that is usually not included in sum-over-states calculations. It represents the double core-polarization by simultaneous action of two external fields; the electric field of laser light and weak electron-nucleus interaction.

This term is negligible for the $6 s-7 s$ PNC transition in Cs by chance only, and is very different for other atoms and transitions. It is $2 \%$ for the $6 s-5 d$ PNC transition of Cs, $5 \%$ for the $7 s-6 d$ transition in $\mathrm{Ra}^{+}$and $40 \%$ for the $6 p_{1 / 2}-6 p_{3 / 2}$ transition in Tl. The last applies to the case when thallium is treated as a one-valence-electron
TABLE IV. Calculated ionisation energies $\left(\mathrm{cm}^{-1}\right)$ including ladder diagrams for $\mathrm{La}^{2+}$ and comparison with experiment.

\begin{tabular}{lcrrrr}
\hline \hline Level & BO & Ladder & Sum & Exp. [19] & \% Diff. \\
\hline $6 s_{1 / 2}$ & 141301 & -204 & 141097 & 141084 & $0.01 \%$ \\
$6 p_{1 / 2}$ & 112930 & -189 & 112741 & 112660 & $0.07 \%$ \\
$6 p_{3 / 2}$ & 109780 & -174 & 109606 & 109564 & $0.04 \%$ \\
$5 d_{3 / 2}$ & 155565 & -1005 & 154561 & 154675 & $-0.07 \%$ \\
$5 d_{5 / 2}$ & 153902 & -1025 & 152877 & 153072 & $-0.13 \%$ \\
\hline \hline
\end{tabular}

TABLE V. Calculated reduced matrix elements of transitions of interest to $s$ - $d$ PNC calculations. RPA equations solved at the energy of each PNC transition. (a.u.)

\begin{tabular}{rlll}
\hline \hline Transition & $\mathrm{Ra}^{+}$ & $\mathrm{Ac}^{2+}$ & $\mathrm{Th}^{3+}$ \\
\hline $7 s_{1 / 2}-7 p_{3 / 2}$ & 4.485 & 3.775 & 3.326 \\
$7 s_{1 / 2}-8 p_{3 / 2}$ & 0.3729 & 0.1755 & 0.0770 \\
$6 d_{3 / 2}-7 p_{1 / 2}$ & 3.533 & 2.569 & 2.100 \\
$6 d_{3 / 2}-8 p_{1 / 2}$ & 0.0440 & 0.2047 & 0.2275 \\
\hline \hline
\end{tabular}


TABLE VI. Calculations of reduced E1 and E2 matrix elements $\left\langle\psi_{f}\left\|\tilde{d}_{E k}\right\| \psi_{i}\right\rangle$ for the Fr-like actinide ions, including Brueckner correlations and core polarization (a.u.).

Note that the reduced matrix elements observe the symmetry property $\langle a|| \hat{d} \| b\rangle=(-1)^{J_{a}-J_{b}}\langle b|| \hat{d} \| a\rangle$.

\begin{tabular}{|c|c|c|c|c|c|c|c|c|}
\hline \multicolumn{2}{|c|}{ E1 } & \multirow[b]{2}{*}{$\mathrm{Fr}$} & \multirow[b]{2}{*}{$\mathrm{Ra}^{+}$} & \multirow[b]{2}{*}{$\mathrm{Ac}^{2+}$} & \multirow[b]{2}{*}{$\mathrm{Th}^{3+}$} & \multirow[b]{2}{*}{$\mathrm{Pa}^{4+}$} & \multirow[b]{2}{*}{$\mathrm{U}^{5+}$} & \multirow[b]{2}{*}{$\mathrm{Np}^{6+}$} \\
\hline$i$ & $f$ & & & & & & & \\
\hline \multirow[t]{4}{*}{$7 s_{1 / 2}$} & $7 p_{1 / 2}$ & 4.287 & 3.228 & 2.707 & 2.377 & 2.147 & 1.969 & 1.830 \\
\hline & $7 p_{3 / 2}$ & 5.906 & 4.482 & 3.771 & 3.316 & 2.995 & 2.745 & 2.546 \\
\hline & $8 p_{1 / 2}$ & 0.287 & -0.071 & -0.196 & -0.261 & -0.306 & -0.342 & -0.370 \\
\hline & $8 p_{3 / 2}$ & 0.890 & 0.360 & 0.148 & 0.026 & -0.060 & -0.131 & - \\
\hline \multirow[t]{4}{*}{$8 s_{1 / 2}$} & $7 p_{1 / 2}$ & -4.195 & -2.497 & -1.887 & -1.554 & -1.340 & -1.188 & -1.101 \\
\hline & $7 p_{3 / 2}$ & -7.425 & -4.621 & -3.563 & -2.974 & -2.595 & -2.329 & -2.162 \\
\hline & $8 p_{1 / 2}$ & 10.150 & 7.006 & 5.599 & 4.758 & 4.186 & 3.762 & 3.426 \\
\hline & $8 p_{3 / 2}$ & 13.442 & 9.386 & 7.547 & 6.437 & 5.676 & 5.107 & 4.643 \\
\hline \multirow[t]{4}{*}{$6 d_{3 / 2}$} & $7 p_{1 / 2}$ & 7.174 & 3.533 & 2.571 & 2.096 & 1.803 & 1.587 & 1.433 \\
\hline & $7 p_{3 / 2}$ & -3.301 & -1.496 & -1.050 & -0.837 & -0.708 & -0.614 & -0.549 \\
\hline & $8 p_{1 / 2}$ & -2.489 & 0.041 & 0.189 & 0.185 & 0.13 & - & - \\
\hline & $8 p_{3 / 2}$ & 0.764 & -0.137 & -0.156 & -0.138 & - & - & - \\
\hline \multirow[t]{3}{*}{$6 d_{5 / 2}$} & $5 f_{7 / 2}$ & -9.117 & -5.666 & -2.819 & -1.930 & -1.498 & -1.217 & -0.996 \\
\hline & $7 p_{3 / 2}$ & 10.156 & 4.795 & 3.417 & 2.749 & 2.344 & 2.044 & 1.835 \\
\hline & $8 p_{3 / 2}$ & -2.499 & 0.379 & 0.487 & 0.453 & 0.366 & - & - \\
\hline \multirow{2}{*}{$5 f_{5 / 2}$} & $6 d_{3 / 2}$ & 7.318 & 4.441 & 2.173 & 1.510 & 1.184 & 0.968 & 0.795 \\
\hline & $6 d_{5 / 2}$ & -2.037 & -1.257 & -0.604 & -0.417 & -0.327 & -0.271 & -0.229 \\
\hline \multicolumn{2}{|c|}{$\overline{\mathrm{E} 2}$} & & & & & & & \\
\hline$i$ & $f$ & $\mathrm{Fr}$ & $\mathrm{Ra}^{+}$ & $\mathrm{Ac}^{2+}$ & $\mathrm{Th}^{3+}$ & $\mathrm{Pa}^{4+}$ & $\mathrm{U}^{5+}$ & $\mathrm{Np}^{6+}$ \\
\hline \multirow[t]{2}{*}{$7 s_{1 / 2}$} & $6 d_{3 / 2}$ & -33.367 & -14.676 & -9.529 & -7.065 & -5.612 & -4.591 & - \\
\hline & $6 d_{5 / 2}$ & -41.568 & -18.868 & -12.373 & -9.225 & -7.359 & -6.049 & -3.9 \\
\hline \multirow{2}{*}{$5 f_{5 / 2}$} & $7 p_{1 / 2}$ & 69.761 & 33.569 & 6.711 & 3.086 & 1.97 & 1.35 & - \\
\hline & $7 p_{3 / 2}$ & -47.191 & -20.289 & -3.408 & -1.438 & - & - & - \\
\hline
\end{tabular}

system, so that the $6 s$ electrons remain in the core. The reason why it is not usually included in sum-over-states calculations is that it cannot be represented as a product of single-electron matrix elements involving valence states. The problem of double core polarization will be considered in more detail elsewhere 21 .

Table II presents our calculated energy levels for Cs, $\mathrm{Ba}^{+}, \mathrm{Fr}$ and $\mathrm{Ra}^{+}$against experimental values. The BO calculations are accurate to around $0.1-0.5 \%$ for most levels, which is typical for this type of calculation. Table II presents our calculated energy levels for the francium-like actinide ions, and Table III shows the percentage difference between our calculations and experimental values for the most important energy intervals for PNC in Fr, $\mathrm{Ra}^{+}, \mathrm{Ac}^{2+}$ and $\mathrm{Th}^{3+}$.

Note that the accuracy can be further improved by including the contributions of the so-called ladder diagrams 22. We illustrate thsi using the $\mathrm{La}^{2+}$ ion as an example. Table IV presents calculations of $\mathrm{La}^{2+}$ ionisation energies including ladder diagrams. The experimental energies are reproduced to an extraordinary accuracy, even for the notoriously difficult $d$ levels. A full inclusion of the ladder diagrams for all ions will be saved for a later work, and currently our method only allows inclusion of ladder diagrams in the energy levels but not PNC. Here we demonstrate that by including the ladder diagrams the accuracy is significantly improved, and that the accuracy in all of these ions is very good.
We also calculate several reduced E1 matrix elements that are of interest to PNC transitions, which are presented in Table V] and in Table VI we present calculations of several of the reduced matrix elements of the considered Fr-like actinide ions.

In francium, the $7 s$ state is the ground state. However, in charged ions this is not necessarily the case. For the ions after actinium the $5 f$ state is pushed below $7 \mathrm{~s}$, forming a new ground state (see TableII). Also, after actinium the $6 d_{3 / 2}$ state is pushed below the $7 s$ state. The ions after $\mathrm{Np}^{6+}$ no longer have closed $p$-shells and are not considered here. A similar crossing of configurations also occurs in the cesium isoelectronic sequence; Cs and $\mathrm{Ba}^{+}$have $6 s$ ground-states, $\mathrm{La}^{2+}$ has $5 d_{3 / 2}$, and $\mathrm{Ce}^{3+}$ and $\mathrm{Pr}^{4+}$ have $4 f$ ground-states (see Tables $\mathrm{I}$ and IV).

For a $7 s-6 d_{3 / 2}$ interval to be a viable transition for the measurement of PNC, one of these states $\left(7 s\right.$ or $\left.6 d_{3 / 2}\right)$ should be either the ground state or a metastable state that can first be populated and then the PNC transition observed.

Also, it was shown it the pivotal work of N Fortson 9 that to ensure accurate PNC measurements of a single trapped ion both the upper and lower levels of the transition should be long lived. In Table VII we present calculations of the lifetimes of the relevent levels for $\mathrm{Ba}^{+}$, $\mathrm{La}^{2+}, \mathrm{Ra}^{+}, \mathrm{Ac}^{2+}$ and $\mathrm{Th}^{3+}$. We show that this condition is met in all of these ions except for $\mathrm{Th}^{3+}$, which has a long-lived upper level but a lower level that quickly 
TABLE VII. Lifetimes (s) of upper and lower states of the $s$ - $d$ PNC transitions for main ions of interest, where $n$ is the principal quantum number: $n=6$ for $\mathrm{Ba}^{+}$and $\mathrm{La}^{2+}, n=7$ for $\mathrm{Ra}^{+}, \mathrm{Ac}^{2+}$ and $\mathrm{Th}^{3+} .(\infty$ means ground state).

\begin{tabular}{llllll}
\hline \hline Level & $\mathrm{Ba}^{+}$ & $\mathrm{La}^{2+}$ & $\mathrm{Ra}^{+}$ & \multicolumn{1}{c}{$\mathrm{Ac}^{2+}$} & $\mathrm{Th}^{3+}$ \\
\hline$(n-1) d_{3 / 2}$ & 84.5 & $\infty$ & 0.642 & $1.19 \times 10^{6}$ & 0.58 \\
$n s_{1 / 2}$ & $\infty$ & 0.347 & $\infty$ & $\infty$ & $1.12 \times 10^{-6}$ \\
\hline \hline
\end{tabular}

decays via E1 transitions.

Note in particular the very long-lived upper $\left(6 d_{3 / 2}\right)$ state of $\mathrm{Ac}^{2+}$. This state is practically stable, the E2 transition back to the $7 \mathrm{~s}$ ground state (the only lower state - see Table II is highly suppressed due to the very small energy gap of this state, $801 \mathrm{~cm}^{-1}$. This is very benefitial for the measurent of PNC in single-trapped ions 9 .

\section{RESULTS AND DISCUSSION}

The final calculations of the $s$ - $d$ and (near) optical $s-s$ PNC amplitudes for the francium-like ions are presented in Table VIII with some previous calculations for comparison. The amplitudes calculated here include corepolarization (RPA) and all-order Brueckner correlations.

For comparison and completeness, these calculations were also performed for $\mathrm{Cs}, \mathrm{Ba}^{+}$and the first few Cslike lanthanide ions. These much lighter ions have correspondingly smaller PNC amplitudes. The results are presented in Table IX. We have not presented a result for the $6 s-7 s$ transition in cesium since this has been investigated thoroughly in our recent work [2].

The PNC amplitudes calculated here agree very well with previous determinations for $\mathrm{Cs}, \mathrm{Ba}^{+}, \mathrm{Fr}$ and $\mathrm{Ra}^{+}$. For $\mathrm{Ra}^{+}$our result is within $1 \%$ of the result calculated in Ref. [8] using the same 'solving equations' method used here [23]. Our $\mathrm{Ra}^{+}$value is also, however, 4-5\% smaller than the amplitudes calculated in that same work as well as in Ref. [24], which used a different 'sum-over-states' approach. The difference is most likely due to the double core-polarization (last term of (3), discussed above), which we calculate to contribute $-4.7 \%$ to this amplitude, and is not included in the sum-over-states calculations. Note that double core-polarization was also not included in our recent calculations for $\mathrm{Ba}^{+}, \mathrm{Yb}^{+}$, and $\mathrm{Ra}^{+}$[27]. This is because in that paper we were focused on the nuclear spin-dependent PNC amplitudes, where high accuracy of the analysis is less important.

The $s d$ PNC transitions tend to have a single dominating term which contributes $\sim 90 \%$ to the total amplitude [8]. In $\mathrm{Th}^{3+}$, for example, this term with the $7 s-7 p_{1 / 2}$ energy interval contributes approximately $96 \%$. The energy interval for this term agrees with experiment to $0.3 \%$ (see Table III). Based on comparison with experimental energies and previous calculations, we expect our amplitudes to be accurate to around 1\%. This accuracy
TABLE VIII. Calculated $7 s-6 d_{3 / 2}$ and $7 s-8 s$ PNC amplitudes for the Fr-like actinide ions, in units $i\left(-Q_{W} / N\right) \times 10^{-11}$ a.u. Also shown are the ground-state levels, experimental wavelengths of the transitions, and several previous PNC calculations for comparison.

\begin{tabular}{|c|c|c|c|c|}
\hline \multirow[t]{2}{*}{ Ion } & \multirow{2}{*}{$\begin{array}{l}\text { ground } \\
\text {-state }\end{array}$} & \multirow[t]{2}{*}{$\lambda(\mathrm{nm})$} & \multicolumn{2}{|c|}{$E_{P N C}$} \\
\hline & & & This work & Others \\
\hline \multirow[t]{3}{*}{${ }^{223} \mathrm{Fr}$} & $7 s_{1 / 2}$ & sd 616 & 57.99 & $57.1(23)$ \\
\hline & & ss 507 & 15.38 & $15.56(17)^{a} 13$ \\
\hline & & & & $15.69(16)^{2} 25$ \\
\hline \multirow[t]{3}{*}{${ }^{226} \mathrm{Ra}^{+}$} & $7 s_{1 / 2}$ & sd 827 & 44.35 & $43.9(17)^{\mathrm{C}}$ \\
\hline & & & & $\begin{array}{ll}46.4 & 10 \\
46.47 \mathrm{a} & 24\end{array}$ \\
\hline & & ss 230 & 10.89 & \\
\hline${ }^{227} \mathrm{Ac}^{2+}$ & $7 s_{1 / 2}$ & sd 12484 & 42.81 & \\
\hline${ }^{232} \mathrm{Th}^{3+}$ & $5 f_{5 / 2}$ & sd 717 & 43.59 & \\
\hline${ }^{231} \mathrm{~Pa}^{4+}$ & $5 f_{5 / 2}$ & sd $324^{\mathrm{d}}$ & 43.49 & \\
\hline${ }^{238} \mathrm{U}^{5+}$ & $5 f_{5 / 2}$ & sd 198 & 45.94 & \\
\hline${ }^{237} \mathrm{~Np}^{6+}$ & $5 f_{5 / 2}$ & $s d 139^{\mathrm{d}}$ & 44.07 & \\
\hline
\end{tabular}

a Contribution of Breit interaction is removed for the convenience of comparison. The final result of $[13$ is -15.41 , and of [24] is 45.89

b Breit and QED corrections removed for comparison. The final result of [25] is 15.49

${ }^{\mathrm{c}}$ Rescaled from ${ }^{223} \mathrm{Ra} 23$.

d Calculated (BO) wavelength

TABLE IX. Calculations of the PNC amplitudes for the Cslike actinide ions and several previous PNC calculations for comparison. In units $i\left(-Q_{W} / N\right) \times 10^{-11}$ a.u.

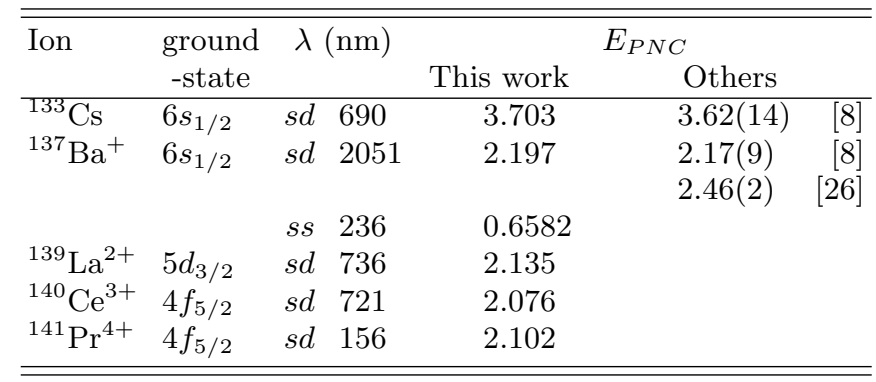

can be improved by including the Breit 28, neutronskin 29] and QED 30] corrections, missed high-order correlations such as ladder diagrams [22] (see Table IV) and structural radiation [18, and with the use of experimental $p$ - $d$ E1 amplitudes. With these corrections the theoretical accuracy can be expected to surpass that of cesium.

The experimental accuracy can be expected to be high due to stable nuclei and large PNC signals. Additionally, in the case of $\mathrm{Ac}^{2+}$ where both upper and lower levels are extremely long-lived, the experimental accuracy has the potential to be very high. 
FIG. 1. Level scheme for $\mathrm{Th}^{3+}$

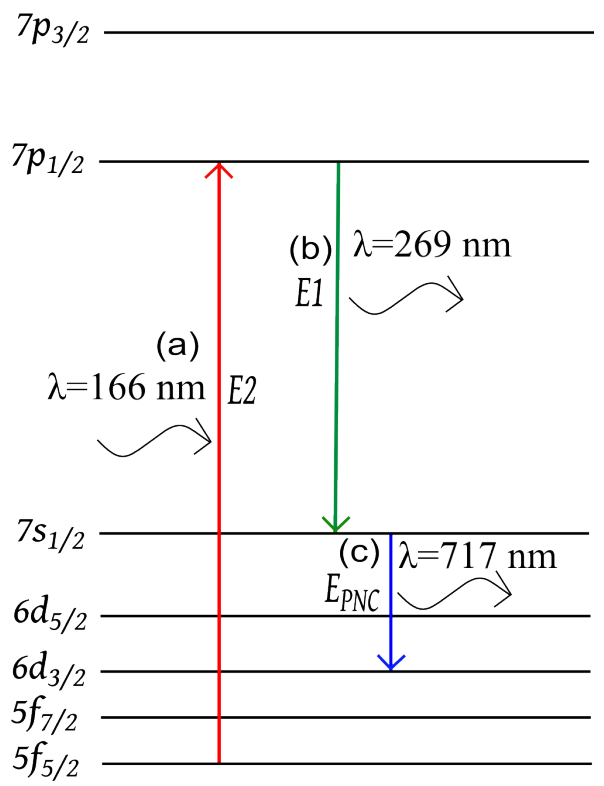

are in very good agreement with existing calculations of Safronova et al. 31, 32. The $7 s$ state should be relatively stable, since there are no allowed E1 transitions to lower states. The only significant contributions to its decay are from E2 transitions to the $6 d_{3 / 2}$ and $6 d_{5 / 2}$ states. We calculate a long lifetime of $0.58 \mathrm{~s}$, in excellent agreement with the recent calculation of M. S. and U. I. Safronova of $0.570(8) \mathrm{s} 32$.

TABLE X. Energies $(\omega)$, probabilities $(\Gamma)$ and per-second transition rates $\left(A_{r}\right)$ of transitions in $\mathrm{Th}^{3+}$.

\begin{tabular}{lllll}
\hline \hline Transition & & $\omega$ (a.u.) & \multicolumn{1}{c}{$\Gamma$ (a.u.) } & $A_{r}\left(\mathrm{~s}^{-1}\right)$ \\
\hline $5 f_{5 / 2}-6 d_{3 / 2}$ & E1 & 0.042 & $1.46 \times 10^{-11}$ & $6.07 \times 10^{5}$ \\
$6 d_{3 / 2}-7 p_{1 / 2}$ & E1 & 0.233 & $7.21 \times 10^{-9}$ & $2.99 \times 10^{8}$ \\
\hline $5 f_{5 / 2}-7 p_{1 / 2}$ & E2 & 0.274 & $3.41 \times 10^{-15}$ & 142 \\
$7 p_{1 / 2}-7 s_{1 / 2}$ & E1 & 0.169 & $7.07 \times 10^{-9}$ & $2.92 \times 10^{8}$ \\
$7 p_{1 / 2}-6 d_{3 / 2}$ & E1 & 0.233 & $1.45 \times 10^{-8}$ & $5.97 \times 10^{8}$ \\
\hline $5 f_{5 / 2}-7 p_{3 / 2}$ & E2 & 0.333 & $1.96 \times 10^{-15}$ & 81.4 \\
$7 p_{3 / 2}-7 s_{1 / 2}$ & E1 & 0.227 & $1.66 \times 10^{-8}$ & $6.90 \times 10^{8}$ \\
$7 p_{3 / 2}-6 d_{3 / 2}$ & E1 & 0.291 & $2.24 \times 10^{-9}$ & $9.28 \times 10^{7}$ \\
$7 p_{3 / 2}-6 d_{5 / 2}$ & E1 & 0.267 & $1.87 \times 10^{-8}$ & $7.30 \times 10^{8}$ \\
\hline $7 s_{1 / 2}-6 d_{3 / 2}$ & E2 & 0.064 & $3.57 \times 10^{-17}$ & 1.48 \\
$7 s_{1 / 2}-6 d_{5 / 2}$ & E2 & 0.039 & $5.57 \times 10^{-18}$ & 0.23 \\
\hline \hline
\end{tabular}

\section{CONCLUSION}

In order to observe the $7 s-6 d_{3 / 2}$ PNC transitions in the actinide ions with the $5 f_{5 / 2}$ ground states, the $7 s$ state must first be populated. In these ions the $5 d_{3 / 2}$ state lies below the $7 s$ state, however it is unstable as it will decay very quickly via an E1 transition to the $5 f_{5 / 2}$ ground state. Population of the $7 \mathrm{~s}$ state can be achieved via optical excitation to the $7 p_{1 / 2}$ or $7 p_{3 / 2}$ levels by a series of E1 transitions (e.g. $5 f-6 d-7 p$ ) or an E2 transition, then $7 p_{1 / 2,3 / 2}$ will spontaneously decay to the $7 s$ state via an E1 transition - see Fig. 1 .

For this method to be viable we need to meet several criteria. First, we need the $7 s$ state to be metastable and have an appropriate lifetime. Second, we should also have that the pumping transition frequencies (to populate $7 s$ ) are in the range of laser spectroscopy. Also, it is necessary that the de-excitation from the $p$ to $s$-level is relatively probable compared to transitions to other levels. If this last condition is not met it is possible to enforce it using stimulated emission, which should not be a problem since these transitions are optical.

Table $\mathrm{X}$ shows the probabilities and per-second transition rates for these transitions in $\mathrm{Th}^{3+}$. Our calculations
We have provided calculations of parity nonconservation, energy levels, matrix elements and lifetimes of several Cs-like rare-earth and Fr-like actinide ions.

We demonstrate that these systems provide a very high theoretical accuracy. With the inclusion of other effects (such as Breit, QED, ladder operators etc.) this could lead to better precision in the calculations than has been achieved in cesium.

With very large PNC amplitudes, these ions can be expected also to have a very high accuracy in the measurements, with the added benefits of (near)-stable nuclei in $\mathrm{Ba}^{+}, \mathrm{La}^{2+}, \mathrm{Ac}^{2+}$ and $\mathrm{Th}^{2+}$, and long-lived upper and lower states for $\mathrm{Ba}^{+}, \mathrm{La}^{2+}, \mathrm{Ra}^{+}$and particularly $\mathrm{Ac}^{2+}$.

\section{ACKNOWLEDGMENTS}

This work was supported by the Australian Research Council.
[1] C. S. Wood, S. C. Bennett, D. Cho, B. P. Masterson, J. L. Roberts, C. E. Tanner and C. E. Wieman, Science 275, 1759 (1997); S. C. Bennett and C. E. Wieman, Phys. Rev. Lett. 82, 2484 (1999); 82, 4153(E) (1999); 83, 889(E) (1999).

[2] V. A. Dzuba, J. C. Berengut, V. V. Flambaum and B. Roberts, Phys. Rev. Lett. 109, 203003 (2012).
[3] S. G. Porsev, K. Beloy and A. Derevianko, Phys. Rev. Lett. 102, 181601 (2009); Phys. Rev. D 82, 036008 (2010).

[4] V. A. Dzuba, V. V. Flambaum and O. P. Sushkov, Phys. Lett. A 141, 147 (1989); V. A. Dzuba, V. V. Flambaum and J. S. M. Ginges, Phys. Rev. D 66, 076013 (2002). 
[5] S. A. Blundell, W. R. Johnson and J. Sapirstein, Phys. Rev. Lett. 65, 1411 (1990); S. A. Blundell, J. Sapirstein and W. R. Johnson, Phys. Rev. D 45, 1602 (1992); M. G. Kozlov, S. G. Porsev and I. I. Tupitsyn, Phys. Rev. Lett. 86, 3260 (2001).

[6] P. G. Blunden, W. Melnitchouk and A. W. Thomas Phys. Rev. Lett. 109, 262301 (2012).

[7] I. B. Khriplovich, Parity nonconservation in atomic phenomena (Gordon and Breach, New York, 1991).

[8] V. A. Dzuba, V. V. Flambaum and J. S. M. Ginges, Phys. Rev. A 63, 062101 (2001).

[9] N. Fortson, Phys. Rev. Lett. 70, 2383 (1993).

[10] L. W. Wansbeek, B. K. Sahoo, R. G. E. Timmermans, K. Jungmann, B. P. Das and D. Mukherjee, Phys. Rev. A 78, 050501 (2008).

[11] E. Gomez, L. A. Orozco and G. D. Sprouse, Rep. Prog. Phys. 69, 79 (2006); D. Sheng, J. Zhang and L. A. Orozco, Rev. Scient. Instr. 83, 043106 (2012); S. Aubin, J. A. Behr, G. Chen, R. Collister, V. V. Flambaum et al., AIP Conf. Proc. 1525, 530 (2013).

[12] V. A. Dzuba, V. V. Flambaum and O. P. Sushkov, Phys. Rev. A 51, 3454 (1995).

[13] M. S. Safronova and W. R. Johnson, Phys. Rev. A 62, 022112 (2000).

[14] J. C. Berengut, V. A. Dzuba, V. V. Flambaum and A. Ong, Phys. Rev. A 86, 022517 (2012).

[15] C. J. Campbell, A. V. Steele, L. R. Churchill, M. V. DePalatis, D. E. Naylor, D. N. Matsukevich, A. Kuzmich and M. S. Chapman, Phys. Rev. Lett. 102, 233004 (2009).

[16] J. S. M. Ginges and V. V. Flambaum, Phys. Rep. 397, $63(2004)$.

[17] V. A. Dzuba, V. V. Flambaum, P. G. Silvestrov and O. P. Sushkov, J. Phys. B 20, 1399 (1987);

[18] V. A. Dzuba, V. V. Flambaum and O. P. Sushkov, Phys. Lett. A 140, 493 (1989). V. A. Dzuba, V. V. Flambaum and O. P. Sushkov, Phys. Lett. A 141, 147 (1989); V. A. Dzuba, V. V. Flambaum, A. Y. Krafmakher and O. P. Sushkov, Phys. Lett. A 142, 373 (1989).

[19] A. Kramida, Yu. Ralchenko, J. Reader and NIST ASD Team. NIST Atomic Spectra Database (2012). Online: http://physics.nist.gov/asd

[20] J. Blaise and J.-F. Wyart, International Tables of Selected Constants (1992). Online: http://www.lac.upsud.fr/Database

[21] B. M. Roberts, V. A. Dzuba and V. V. Flambaum, to be published.

[22] V. A. Dzuba, Phys. Rev. A 78, 042502 (2008).

[23] The information about isotope numbers is missed in our old paper 8 . It is most likely ${ }^{223} \mathrm{Ra}$ in the mixed states calculations and ${ }^{226} \mathrm{Ra}$ in the sum-over-states calculations.

[24] R. Pal, D. Jiang, M. S. Safronova and U. I. Safronova, Phys. Rev. A 79, 062505 (2009).

[25] V. M. Shabaev, I. I. Tupitsyn, K. Pachucki, G. Plunien and V. A. Yerokhin, Phys. Rev. A 72, 062105 (2005).

[26] B. K. Sahoo, B. P. Das, R. K. Chaudhuri and D. Mukherjee, Phys. Rev. A 75, 032507 (2007).

[27] V. A. Dzuba and V. V. Flambaum, Phys. Rev. A 83, 052513 (2011).

[28] V. A. Dzuba, V. V. Flambaum and M. S. Safronova, Phys. Rev. A, 73022112 (2006).

[29] A. Derevianko, Phys. Rev. A 65, 012106 (2001).

[30] B. M. Roberts, V. A. Dzuba and V. V. Flambaum, Phys. Rev. A 87, 054502 (2013).

[31] U. I. Safronova, W. R. Johnson and M. S. Safronova, Phys. Rev. A 74, 042511 (2006).

[32] M. S. Safronova and U. I. Safronova, arXiv:1304.0158 (2013). 